\title{
Impactos da pandemia da COVID-19 em idosos e estratégias de reabilitação
}

\section{adotadas}

\author{
Impacts of the COVID-19 pandemic on elderly and adopted rehabilitation clinics \\ Impactos de la pandemia COVID-19 en los ancianos y estrategias de rehabilitación adoptadas
}

Recebido: 02/12/2021 | Revisado: 07/12/2021 | Aceito: 14/12/2021 | Publicado: 21/12/2021

Ester Miranda de Sousa

ORCID: https://orcid.org/0000-0003-2319-4840 Faculdade de Ensino Superior do Piauí, Brasil E-mail: mirandaester70@gmail.com

Ivisson Lucas Campos da Silva

ORCID: https://orcid.org/0000-0003-2848-9402 Faculdade de Ensino Superior do Piauí E-mail: ivissonlucas@hotmail.com

Luigi Gabriel Brasil da Silva

ORCID: https://orcid.org/0000-0002-8556-3958

Faculdade de Ensino Superior do Piauí,brasil E-mail: luigigabrie1010@outlook.com

José Edmilson da Silva Neto

ORCID: https://orcid.org/0000-0002-6372-0481

Faculdade de Ensino Superior do Piauí, Brasil

E-mail: edmilson161214@ @otmail.com

Diane Nogueira Paranhos Amorim

ORCID: https://orcid.org/0000-0002-5362-5747

Faculdade de Ensino Superior do Piauí, Brasil

E-mail: diannenogueira@hotmail.com

Ruth Raquel Soares de Farias

ORCID: https://orcid.org/0000-0002-0988-0900

Faculdade de Ensino Superior do Piauí, Brasil E-mail: ruthraquelsf@gmail.com

Francisco Maurilio da Silva Carrias

ORCID: https://orcid.org/0000-0002-0762-0494

Faculdade de Ensino Superior do Piau

E-mail: mauriliocarrias@gmail.com

Lélia Lilianna Borges de Sousa Macedo

ORCID: https://orcid.org/0000-0003-1108-6324

Faculdade de Ensino Superior do Piauí

E-mail: leliafisio@hotmail.com

\begin{abstract}
Resumo
Introdução: O mundo atualmente enfrenta uma pandemia que modificou os hábitos de vida da população em geral e gerou consequências na economia e na saúde pública, tendo em vista a potencial disseminação do patógeno, o SARS$\mathrm{CoV}-2$, que causa a doença COVID-19. As pessoas maiores de 60 anos foram apontadas como um dos grupos mais vulneráveis a desenvolverem a forma grave da doença. O objetivo deste estudo foi verificar na literatura os impactos da pandemia da COVID-19 em idosos e as estratégias de reabilitação que vêm sendo adotadas neste período. Metodologia: Tratou-se de uma revisão integrativa que utilizou artigos indexados nas bases de dados Medline, PubMed e Scielo publicados entre os anos 2020 e 2021 nos idiomas inglês, português e espanhol. Resultados: A pandemia da COVID-19 e o isolamento social trouxeram alguns transtornos para a saúde do idoso. Os artigos analisados nesta revisão elencaram alguns destes transtornos, entre eles função motora prejudicada, comprometimento na realização das atividades de vida diária, fraqueza adquirida na UTI e piora da fragilidade. Considerações Finais: A saúde do idoso no cenário atual demanda uma atenção mais específica que busque enfatizar não só a recuperação, como também a prevenção desses agravos à saúde. Além disso, as estratégias para alcançar esses objetivos devem ser melhor estudadas, a fim de encontrar métodos para o fornecimento de assistência de qualidade a essa população durante e no pós-pandemia.
\end{abstract}

Palavras-chave: Idosos; COVID-19; Atividades da vida diária; Reabilitação funcional.

\begin{abstract}
Introduction: The world is currently facing a pandemic that has changed the lifestyle of the population in general and generated consequences for the economy and public health, given the potential spread of the pathogen, SARS-CoV-2, which causes the disease COVID- 19. People over 60 years of age were identified as one of the most vulnerable
\end{abstract}


groups to develop the severe form of the disease. The aim of this study was to verify in the literature the impacts of the COVID-19 pandemic on the elderly and the rehabilitation strategies that have been adopted in this period. Methodology: This was an integrative review that used articles indexed in Medline, PubMed and Scielo databases published between the years 2020 and 2021 in English, Portuguese and Spanish. Results: The COVID-19 pandemic and social isolation brought some health problems to the elderly. The articles analyzed in this review listed some of these disorders, including impaired motor function, impairment in performing activities of daily living, weakness acquired in the ICU and worsening of frailty. Final Considerations: The health of the elderly in the current scenario demands more specific attention that seeks to emphasize not only recovery, but also the prevention of these health problems. Furthermore, strategies to achieve these goals should be further studied in order to find methods to provide quality care to this population during and post-pandemic.

Keywords: Seniors; COVID-19; Activities of daily living; Functional rehabilitation.

\section{Resumen}

Introducción: El mundo se enfrenta actualmente a una pandemia que ha cambiado el estilo de vida de la población en general y ha generado consecuencias para la economía y la salud pública, dada la potencial propagación del patógeno, SARS-CoV-2, causante de la enfermedad COVID-19. Las personas mayores de 60 años fueron identificadas como uno de los grupos más vulnerables para desarrollar la forma severa de la enfermedad. El objetivo de este estudio fue verificar en la literatura los impactos de la pandemia COVID-19 en las personas mayores y las estrategias de rehabilitación que se han adoptado en este período. Metodología: Se trata de una revisión integradora que utilizó artículos indexados en las bases de datos Medline, PubMed y Scielo publicados entre los años 2020 y 2021 en inglés, portugués y español. Resultados: La pandemia de COVID-19 y el aislamiento social trajeron algunos problemas de salud a los ancianos. Los artículos analizados en esta revisión enumeraron algunos de estos trastornos, que incluyen la función motora deteriorada, el deterioro en el desempeño de las actividades de la vida diaria, la debilidad adquirida en la UCI y el empeoramiento de la fragilidad. Consideraciones Finales: La salud de las personas mayores en el escenario actual demanda una atención más específica que busca enfatizar no solo la recuperación, sino también la prevención de estos problemas de salud. Además, las estrategias para lograr estos objetivos deben estudiarse más a fondo con el fin de encontrar métodos para brindar una atención de calidad a esta población durante y después de una pandemia.

Palabras clave: Personas mayores; COVID-19; Actividades de la vida diaria; Rehabilitación funcional.

\section{Introdução}

O Vírus da Síndrome Respiratória Aguda Grave Coronavírus-2 (SARS-CoV-2) modificou os hábitos de vida da população em nível mundial e gerou consequências na economia e na saúde pública, tendo em vista o alcance territorial e a velocidade de disseminação do patógeno. Desde o primeiro surto de pneumonia relacionado ao coronavírus em dezembro de 2019, o vírus se deslocou para além das fronteiras chinesas e atingiu mais de 210 países. Segundo a Organização Mundial de Saúde (OMS), em janeiro de 2021, mais de 83 milhões de indivíduos no mundo tiveram infecção confirmada por SARS-CoV2 com base em testes de detecção molecular e mais de 1,8 milhão de mortes foram atribuídas à Corona Vírus Disease (COVID19), doença causada pelo novo coronavírus.

Ademais, as pessoas maiores de 60 anos foram apontadas como um dos grupos mais vulneráveis no início da pandemia, pois quanto mais avançada a idade e debilitado for o organismo, maior a propensão a sequelas da infecção pelo coronavírus e maior o risco de desenvolver o que se chama de síndrome pós-Covid. Uma vez que o vírus afetou amplamente os idosos, as medidas de bloqueio para essa população tiveram de ser mais rígidas, desse modo, as consequências e os efeitos colaterais também foram intensificados nessa população (Van Jaarsveld, 2021).

Em princípio, uma das medidas de prevenção e proteção à saúde adotada foi o isolamento social que traz como consequência a elevação do risco de problemas cardiovasculares, autoimunes, cognitivos e de saúde mental, como por exemplo a desconexão social que expõe os idosos a um maior risco de depressão, ansiedade e declínio funcional (Santini et al., 2020), a julgar por este grupo populacional estar susceptível a complicações mais graves, cujos desfechos podem resultar em óbito. Relatórios iniciais mostraram que aproximadamente $80 \%$ das mortes devido ao COVID-19 ocorrem em pessoas com mais de 65 anos (CDC, 2020). 
Conforme a pessoa envelhece, a perda de capacidades físicas e mentais é notório para todos, embora esse processo natural do envelhecimento humano ocorra de maneira diferente entre os indivíduos. Tais problemas provavelmente serão agravados pelas limitações físicas impostas ao movimento dos indivíduos fora de suas casas, resultando em menos oportunidades de exercícios para muitos indivíduos. A perda de socialização, aumento da tensão mental e problemas gerais de saúde mental e diminuição do exercício podem ter efeitos negativos substanciais nos idosos (Van Jaarsveld, 2021).

Tendo em vista o surgimento de um descondicionamento muscular dos membros inferiores e de um comprometimento do desempenho das Atividades de Vida Diária (AVDs), os pacientes com COVID-19 que sofrem de inúmeros sintomas durante o período de hospitalização em UTI, e que requerem tratamento agudo em um estrito grau de isolamento, terão sua mobilidade reduzida (Steardo et al., 2020). Logo, é verídico afirmar que os idosos com COVID-19 precisem de intervenções diretas de reabilitação após a admissão hospitalar.

A dependência tende a se acentuar em todas as faixas etárias, sobretudo na população idosa, após longas permanências na UTI (Herridge et al., 2016). De modo que, prejuízos funcionais no organismo, como dispneia, fraqueza, mialgia e outras sensações de dor, levam a uma limitação da capacidade de realizar as AVDs. De acordo com Zerah et al. (2020), ao analisar as características clínicas e resultados de 821 pacientes idosos com infecção por SARS-CoV-2 internados em enfermarias geriátricas, observou uma redução da independência nas AVDs associada a um pior prognóstico após a COVID-19.

Assim sendo, o conhecimento sobre os aspectos relacionados à saúde do idoso inerentes à pandemia de COVID-19, exerce um diferencial para tomada de decisões coerentes por órgãos públicos mediante a proteção e preservação da saúde deste grupo etário. Apesar do isolamento social ainda ser a opção mais recomendada pela OMS com o intuito de reduzir a velocidade de contaminação e minimizar a sobrecarga nos sistemas de saúde, essa medida pode intensificar a inatividade física e o sedentarismo (Hall et al., 2020). Logo, o isolamento social contribui para o surgimento de doenças musculoesqueléticas, como artrite reumatoide, espondilites e lúpus eritematoso sistêmico, as quais estão associadas a quadros de dores miofasciais e artralgias.

Nesse contexto, a fisioterapia poderá contribuir seguindo normas padronizadas impostas para combater a disseminação do SARS-CoV-2 e evitar a contaminação entre indivíduos da população, sobretudo dos idosos, de forma a integrar programas que envolvam exercícios e estratégias durante a quarentena, que possam integrar a manutenção de um comportamento de estilo de vida positivo, estimulação cognitiva e realização de atividade física, articuladas a técnicas de relaxamento que envolvam respiração diafragmática ou relaxamento muscular. Atuando também no contexto da reabilitação, a fisioterapia é imprescindível na recuperação ou em minimizar os efeitos deletérios da pandemia.

Diante do que foi exposto, o objetivo deste estudo foi verificar na literatura os impactos da pandemia da COVID-19 em idosos e as estratégias de reabilitação adotadas neste período.

\section{Metodologia}

Tratou-se de uma revisão de literatura do tipo integrativa. A revisão integrativa da literatura é um método que tem a finalidade de sintetizar resultados obtidos em pesquisas sobre um delimitado tema ou questão, de maneira sistemática e ordenada, com o objetivo de contribuir para o conhecimento desse tema ou questão (Roman; Friedlander, 1998). Utilizou-se as bases de dados: Medline, Lilacs e PubMed. A pesquisa foi realizada no período de agosto a outubro de 2021 e teve como questão norteadora: "Quais os impactos da pandemia da COVID-19 nos idosos e quais estratégias de reabilitação utilizadas?"

Durante a pesquisa foram avaliados trabalhos com títulos e resumos que se referem ao tema. Utilizou-se os seguintes descritores: "Idosos", "COVID-19", "Atividades de Vida Diária” e "Reabilitação Funcional". A estratégia de busca está explícita no Fluxograma 1. Os critérios de inclusão adotados foram: ensaios clínicos controlados randomizados, estudos 
observacionais e estudos de caso publicados entre os anos 2020 e 2021 nos idiomas inglês, português e espanhol. Os critérios de exclusão foram: monografias, teses, dissertações, editoriais e artigos publicados em revistas não indexadas.

A busca de artigos foi realizada por dois revisores independentes utilizando os seguintes critérios: leitura do título, em seguida leitura dos resumos e posteriormente leitura completa dos artigos. Após a leitura dos artigos na íntegra, foram excluídos aqueles que não obedeceram aos critérios de inclusão e os artigos selecionados foram expostos em um quadro.

\section{Resultados}

Ao todo, foram encontrados 75 artigos nas bases de dados pesquisadas, destes, seis foram incluídos. O Fluxograma 1 detalha o processo de inclusão e exclusão dos artigos.

Fluxograma 1: Fluxograma de inclusão e exclusão dos artigos.

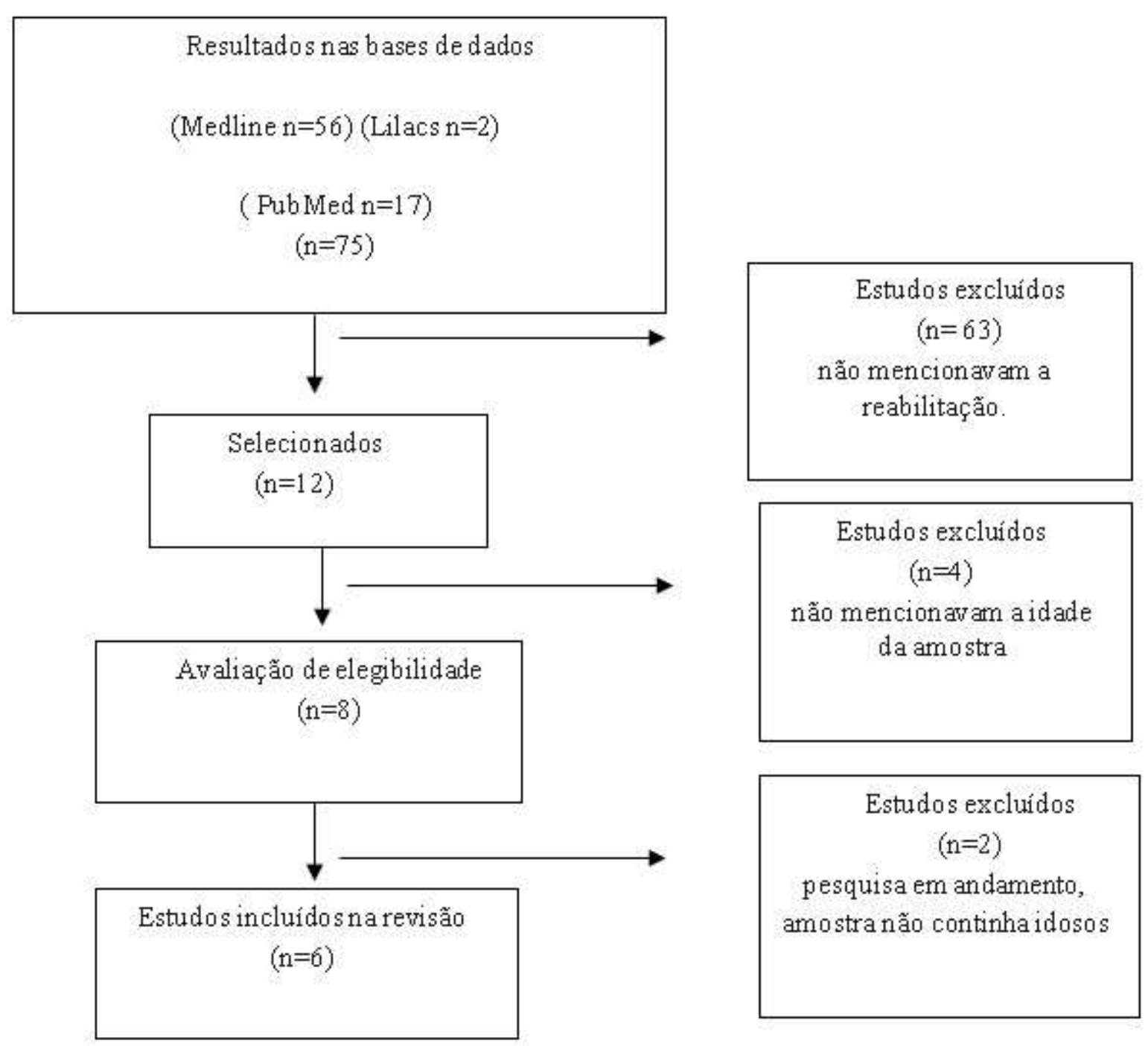

Fonte: Autores.

As informações detalhadas dos artigos incluídos estão expostas no Quadro 1, a seguir. Dos seis trabalhos incluídos, dois citaram fragilidade e comprometimento físico nos idosos, um artigo citou que a fisioterapia se mostrou eficaz na melhora da mobilidade de idosos hospitalizados. Outro artigo traz que a reabilitação no ambiente hospitalar é eficaz e pode melhorar o estado funcional dos idosos internados com COVID-19. 
Tabela 1. Caracterização dos artigos.

\begin{tabular}{|c|c|c|c|c|c|}
\hline $\begin{array}{c}\text { Autor/ } \\
\text { Ano }\end{array}$ & Título & Objetivo & $\begin{array}{c}\text { Tipo de estudo/ } \\
\text { Amostra } \\
\end{array}$ & Protocolo & $\begin{array}{c}\text { Principais } \\
\text { Resultados/ Conclusão }\end{array}$ \\
\hline $\begin{array}{l}\text { Belli, Balbi } \\
\text { \& Prince } \\
(2020)\end{array}$ & $\begin{array}{l}\text { Low physical functioning } \\
\text { and impaired performance } \\
\text { of activities of daily life in } \\
\text { COVID-19 patients who } \\
\text { survived hospitalization. }\end{array}$ & $\begin{array}{l}\text { Avaliar a proporção de } \\
\text { pacientes idosos com } \\
\text { baixo funcionamento } \\
\text { físico e / ou } \\
\text { desempenho } \\
\text { prejudicado de AVDs } \\
\text { na alta hospitalar. }\end{array}$ & $\begin{array}{l}\text { Estudo transversal } \\
\text { retrospectivo/ } \\
103 \text { pacientes } \\
(51,5 \% \text { homens, } \\
\text { idade } 73,9 \pm 12,9 \\
\text { anos. }\end{array}$ & \begin{tabular}{lr}
\multicolumn{3}{l}{ Análise dos } & dados \\
de rotina & de \\
pacientes & com \\
COVID-19 & \\
internados em & um \\
instituto & de \\
reabilitação & na \\
Itália. & \\
\end{tabular} & $\begin{array}{l}\text { Os idosos pós-COVID-19 podem ter } \\
\text { funcionamento físico prejudicado quando } \\
\text { recebem alta, mesmo após mobilização } \\
\text { precoce à beira do leito. }\end{array}$ \\
\hline $\begin{array}{l}\text { Jain, } \\
\text { Harmon \& } \\
\text { Sonagere } \\
(2021)\end{array}$ & $\begin{array}{l}\text { Functional outcomes and } \\
\text { post-discharge care sought } \\
\text { by patients with COVID- } \\
19 \text { compared to matched } \\
\text { controls after completing } \\
\text { inpatient } \\
\text { rehabilitation. }\end{array}$ & $\begin{array}{l}\text { Examinar a eficácia da } \\
\text { reabilitação aguda em } \\
\text { idosos e comparar seus } \\
\text { resultados com os de } \\
\text { pacientes não COVID- } \\
19 \text { apresentando na } \\
\text { mesma idade e grupo } \\
\text { de deficiência. }\end{array}$ & $\begin{array}{lr}\text { Estudo de } & \text { coorte } \\
\text { prospectivo/ } & 36 \\
\text { pacientes } & (18 \\
\text { pacientes } & \text { com } \\
\text { COVID-19 e } & 18 \\
\text { controles) com } \\
\text { idade acima de } 64 \\
\text { anos. }\end{array}$ & $\begin{array}{l}\text { A coleta de dados } \\
\text { foi feita a partir } \\
\text { das respostas dos } \\
\text { pacientes a um } \\
\text { questionário } \\
\text { administrado após } \\
\text { a alta via telefone. }\end{array}$ & $\begin{array}{l}\text { os pacientes da coorte COVID-19 } \\
\text { atingiram níveis de independência } \\
\text { funcional na alta, semelhantes aos de seus } \\
\text { controles pareados. }\end{array}$ \\
\hline $\begin{array}{l}\text { Johnson et } \\
\text { al. }(2021)\end{array}$ & $\begin{array}{l}\text { Frequency of Physical } \\
\text { Therapist Intervention Is } \\
\text { Associated With Mobility } \\
\text { Status and Disposition at } \\
\text { Hospital Discharge for } \\
\text { Patients With COVID-19. }\end{array}$ & $\begin{array}{l}\text { Examinar a relação da } \\
\text { frequência e duração } \\
\text { da visita de } \\
\text { fisioterapia no hospital } \\
\text { com o estado de } \\
\text { mobilidade dos } \\
\text { pacientes na alta e } \\
\text { probabilidade de alta } \\
\text { para casa. }\end{array}$ & $\begin{array}{l}\text { Estudo } \\
\text { retrospectivo/ } 312 \\
\text { pacientes } \\
\text { internados com } \\
\text { COVID-19 com } \\
\text { idade média de } \\
69.6 \text { anos. }\end{array}$ & $\begin{array}{l}\text { Os dados foram } \\
\text { extraídos da } \\
\text { Cleveland Clinic } \\
\text { Health Sistema de } \\
\text { registro de dados } \\
\text { de pesquisa } \\
\text { COVID-19 }\end{array}$ & $\begin{array}{l}\text { Para pacientes com COVID-19, a } \\
\text { fisioterapia realizada com mais frequência } \\
\text { no hospital está diretamente relacionada a } \\
\text { melhores mobilidades na alta e maior } \\
\text { probabilidade de alta. }\end{array}$ \\
\hline $\begin{array}{l}\text { Gwak \& } \\
\text { Hwang } \\
(2021)\end{array}$ & $\begin{array}{l}\text { Step-by-step inpatient } \\
\text { rehabilitation for critical } \\
\text { illness after coronavirus } \\
\text { disease } 2019\end{array}$ & $\begin{array}{l}\text { Apresentar um caso de } \\
\text { reabilitação após } \\
\text { indução de COVID-19 } \\
\text { grave }\end{array}$ & $\begin{array}{l}\text { Estudo de caso/ } \\
1 \text { paciente do sexo } \\
\text { masculino de } 74 \\
\text { anos } \quad \text { com } \\
\text { COVID-19 }\end{array}$ & $\begin{array}{l}\text { Apresentação de } \\
\text { caso }\end{array}$ & $\begin{array}{l}\text { A reabilitação para idosos com COVID-19 } \\
\text { é eficaz para a recuperação funcional e } \\
\text { pode ser realizado com segurança na UTI } \\
\text { e na enfermaria geral. }\end{array}$ \\
\hline $\begin{array}{l}\text { Trevissón- } \\
\text { Redondo et } \\
\text { al. }(2021)\end{array}$ & $\begin{array}{l}\text { USE OF THE BARTHEL } \\
\text { INDEX TO ASSESS } \\
\text { ACTIVITIES OF DAILY } \\
\text { LIVING BEFORE AND } \\
\text { AFTER SARS-COVID } 19 \\
\text { INFECTION } \\
\text { INSTITUTIONALIZED } \\
\text { NURSING } \\
\text { PATIENTS }\end{array}$ & $\begin{array}{l}\text { Avaliar as atividades } \\
\text { de vida diária (AVDs) } \\
\text { antes e após a COVID- } \\
19 \text { e também } \\
\text { determinar se os } \\
\text { resultados variaram ou } \\
\text { não de acordo com o } \\
\text { gênero. }\end{array}$ & $\begin{array}{lr}\text { Estudo de coorte } \\
\text { prospectivo } \\
\text { longitudinal. } \\
\text { pacientes } \\
\text { geriátricos } \\
\text { coabitantes, } \\
\text { homens e } & \\
\text { mulheres } & 34 \\
\text { idade média de } \\
85,86 \pm 6,42 .\end{array}$ & $\begin{array}{l}\text { As AVDs foram } \\
\text { avaliadas nos } \\
\text { residentes } \\
\text { periodicamente } \\
\text { pela enfermeira } \\
\text { por meio do índice } \\
\text { de Barthel. }\end{array}$ & $\begin{array}{l}\text { Houve redução significativa da qualidade } \\
\text { de vida dos idosos nas AVDs. .A } \\
\text { deterioração das AVDs foi mais evidente } \\
\text { nos homens do que nas mulheres }\end{array}$ \\
\hline $\begin{array}{l}\text { Shinohara } \\
\text { et al. } \\
(2021)\end{array}$ & $\begin{array}{lr}\text { ACTUAL } & \text { FRAILTY } \\
\text { CONDITIONS } & \text { AND } \\
\text { LIFESTYLE } & \text { CHANGES } \\
\text { IN COMMUNITY- } \\
\text { DWELLING } & \text { OLDER } \\
\text { ADULTS AFFECTED BY } \\
\text { CORONAVIRUS } \\
\text { DISEASE } \\
\text { COUNTERMEASURES } \\
\text { IN JAPAN: A CROSS- } \\
\text { SECTIONAL STUDY } \\
\end{array}$ & $\begin{array}{l}\text { Esclarecer as reais } \\
\text { condições } \\
\text { fragilidade e mudanças } \\
\text { no estilo de vida entre } \\
\text { idosos residentes na } \\
\text { comunidade afetados } \\
\text { pelas contramedidas } \\
\text { COVID-19 no Japão. }\end{array}$ & $\begin{array}{l}\text { Estudo transversal } \\
\text { realizado com } \\
1.078 \text { idosos com } \\
\text { idade } \geq 65 \text { anos. }\end{array}$ & $\begin{array}{l}\text { Questionários } \\
\text { autorreferidos; } \\
\text { índice de triagem } \\
\text { de fragilidade e o } \\
\text { Questionário para } \\
\text { Mudança de Vida }\end{array}$ & $\begin{array}{l}\text { Dos } 680 \text { idosos }(63,1 \%) \text { analisados, } 60 \\
(8,8 \%) \text { apresentavam fragilidade e } 354 \\
(52,1 \%) \text { pré-fragilidade. O estado de } \\
\text { fragilidade foi significativamente } \\
\text { associado às mudanças no estilo de vida }\end{array}$ \\
\hline
\end{tabular}

Fonte: Autores.

\section{Discussão}

A pandemia da COVID-19 trouxe consigo alguns transtornos para a saúde da pessoa idosa. O distanciamento social trouxe consequências negativas para a saúde física de idosos, ocasionados principalmente pela diminuição dos níveis de atividade física devido à restrição total ou parcial da participação social em grupos comunitários e atividades familiares. Os artigos analisados nesta revisão, elencaram de forma vasta, alguns destes transtornos que afetaram de forma considerável a vida dos idosos em todo o mundo. Função motora prejudicada, comprometimento na realização das AVDs, fraqueza adquirida na UTI e piora da fragilidade foram alguns dos impactos mais citados pelos autores.

O estudo retrospectivo realizado por Belli et al. (2020) que contou com uma amostra de 103 idosos diagnosticados com COVID-19, relatou que $88 \%$ dos idosos que foram afetados pelo SARS CoV-2 tinham duas ou mais comorbidades e 55\% 
apresentavam quatro ou mais. Muitos pacientes relataram dispneia, tosse, febre (90\%), depressão (33\%), disfagia (16\%) e/ou comprometimento cognitivo $(33 \%)$.

Esses achados corroboram com o estudo de Steardo et al. (2020) no qual os pacientes com COVID-19 tiveram múltiplos sintomas durante a hospitalização e os cuidados agudos ocorreram em estrito isolamento, o que reduziu a mobilidade do paciente para zero tornando os idosos mais propensos ao descondicionamento muscular dos membros inferiores e um desempenho prejudicado das atividades da vida diária.

Isto sugere que a prevenção deve ser focada nos idosos para ofertar uma melhor promoção de saúde a esses indivíduos. Essa afirmação é corroborada pela Portaria nº 2.528, de 19 de outubro de 2006, que aprovou a Política Nacional de Saúde da Pessoa Idosa, que destaca a importância de recuperar, manter e promover a autonomia e a independência dos idosos, direcionando medidas coletivas e individuais para este fim.

Além do mais, a participação social possui inúmeros efeitos positivos na saúde física dos idosos (Tomioka et al.,), que envolvem o estímulo dos sistemas musculoesquelético, cardiovascular, respiratório e nervoso (Fernandez-Alonso et al., 2016). Equilíbrio dinâmico e força muscular, função pulmonar saudável e menores deficiências e inflamação crônica em comparação com aqueles sem participação social são alguns dos benefícios que a atividade física regular traz com o intuito de contribuir para um envelhecimento mais saudável (Tomioka et al., 2017; Sepúlveda-Loyola et al., 2020).

O estudo de Belli et al. (2020) compila ainda que 78\% dos idosos diagnosticados com COVID-19, que realizaram um teste de aptidão física, estavam no grupo de baixo desempenho e 67\% dos idosos apresentaram dependência funcional e 45,6\% estavam acamados. Dessa forma, os idosos pós COVID-19 têm uma clara necessidade de intervenções reabilitadoras.

Jain et al. (2021) ratificam esses achados, no qual a maioria dos pacientes apresentou debilidade e, por isso, tiveram um tempo de internação mais longo e necessitaram de suporte de oxigênio durante a fase aguda da internação. Um dado importante desse estudo foi que $94 \%$ dos idosos apresentaram disfunção cognitiva com comprometimento leve a moderado; nervosismo e ansiedade também foram documentados com mais frequência nos idosos com COVID-19. Dessa forma a disfunção cognitiva foi identificada como uma potencial sequela dessa doença. Todavia, no estudo de Curci et al. (2021), os pacientes no momento da admissão na unidade de reabilitação COVID-19 mostraram habilidades cognitivas preservadas.

Kato et al. (2021) em seu estudo que apresenta casos de idosos que tiveram a reabilitação cardíaca interrompida em virtude da pandemia, relatam que o desempenho físico foi prejudicado ocasionando piora da fragilidade nesses idosos e que embora o estado de fragilidade não tenha mostrado melhora significativamente após três meses de retomada da reabilitação, houve uma tendência de melhora desse estado entre os idosos cujo nível de atividade física aumentou.

Sabe-se que a atividade física produz benefícios para a saúde física da população idosa, de forma que estimula a contração muscular, gera gasto de energia, diminui a inflamação sistêmica e o estresse oxidativo, reduz a prevalência de doenças crônicas e síndromes geriátricas como sarcopenia, osteosarcopenia e fragilidade (Arazi \& Eghbali, 2018). Logo, manter um estilo de vida ativo em casa é importante para a saúde dos idosos, principalmente daqueles com doenças crônicas e síndromes geriátricas.

Embora muitos estudos tenham identificado fatores de risco que parecem predispor os idosos a experimentar doença mais grave de COVID-19, ainda não é totalmente entendível quais idosos com esses fatores de risco irão progredir para desenvolver doença grave (Jain et al., 2021). Segundo Johnson et al. (2020), há uma necessidade de entender melhor as complicações funcionais pós agudas relacionadas à COVID-19 e criar soluções para resolver quaisquer déficits persistentes.

Em relação à saúde mental, as mudanças no estilo de vida decorrentes da pandemia foram associadas a resultados adversos para a saúde mental. Segundo Shahid et al. (2020) e Gerwen et al. (2021) houve um aumento de sintomas de depressão, ansiedade e estresse, e um declínio geral da saúde mental durante a quarentena. Estudo realizado por SepúlvedaLoyola et al. (2020), abordando os efeitos mentais e físicos advindos do impacto do isolamento social de idosos por conta da 
COVID-19, encontraram a prevalência de ansiedade e depressão, tendo uma ampla variação de 8,3\% a 49,7\% para ansiedade ou $14,6 \%$ a 47,2\% para depressão, em consonância com estudos anteriores de outras epidemias.

Ademais, a atividade física tem sido associada a melhores resultados psicológicos e seus efeitos podem influenciar mecanismos neurais relacionados aos transtornos depressivos e de ansiedade. Silva et al. (2020), corrobora tal informação em seu estudo sobre inatividade física associada a níveis elevados de ansiedade, depressão e estresse em brasileiros durante a pandemia de COVID-19. A ativação de neurotransmissores, como dopamina e serotonina, a liberação de endorfinas e o processo de termogênese decorrentes da atividade física reduzem o estresse e melhoram o estado de humor (Mello et al., 2005).

Shinohara et al. (2021) analisaram as condições de fragilidade e mudanças no estilo de vida entre idosos residentes na comunidade afetados pelas contramedidas da COVID-19 no Japão e evidenciaram que nos participantes com fragilidade, a quantidade de movimento diário e a força muscular das pernas diminuíram significativamente, enquanto a preocupação ou a ansiedade aumentaram significativamente.

Ademais, sabe-se que o sedentarismo está associado a vários resultados negativos para a saúde e aumenta o risco de quedas, fraturas e incapacidades em idosos. É necessário implementar exercícios físicos (como caminhada, resistência à baixa carga ou exercícios na cama) durante os períodos de desuso para proteger a massa muscular e a função da crise catabólica, visto que os idosos têm uma resposta embotada à reabilitação física, sendo necessária uma combinação de intenso treinamento de resistência e nutrição para superar a perda de músculo esquelético devido ao desuso (Moro \& Paoli, 2020). Bull et al. (2020) acrescenta que as diretrizes atuais sugerem que os idosos devem melhorar sua função física realizando atividades destinadas a melhorar ou manter a força muscular, equilíbrio e atividades de flexibilidade em pelo menos dois dias por semana.

Cunningham e O'Sullivan (2020) corroboram essa afirmação ao alegar que as restrições de saúde pública em vigor para prevenir a propagação da transmissão tiveram impacto sobre os níveis de atividade física, sobretudo nos idosos, os quais tem dificuldades para atingir os níveis recomendados de atividade física. Em relação aos idosos que tiveram tratamentos interrompidos e ficaram em confinamento devido a pandemia, Kato et al. (2021) sugerem que a prática de exercícios alternativos em casa é necessária para evitar a diminuição da atividade física e o desenvolvimento de fragilidade nos idosos e que o aumento da atividade física pode estar associado à melhora da fragilidade após um estado de emergência.

Para Belli et al. (2020) a fisioterapia foi crucial durante a internação pós aguda, no entanto o funcionamento físico e o desempenho das AVDs ainda eram significativamente prejudicados na alta hospitalar. A reabilitação pós hospitalização demonstrou ser benéfica e segura resultando em melhor funcionamento físico e qualidade de vida em pacientes que já tinham como base a doença pulmonar obstrutiva crônica. Apenas 12, dos 103 pacientes desse estudo foram ventilados mecanicamente, isso sugere que pacientes com COVID-19 hospitalizados sem ventilação mecânica também devem ter reabilitação pós hospitalar.

Fan et al. (2014) afirma que a fraqueza muscular é considerada um dos sintomas mais perceptíveis em pacientes com longos períodos acamados na UTIs. Isso parece importante porque, de acordo com Parry e Puthucheary (2015) o número de dias de repouso no leito durante a hospitalização ou permanência na UTI é hoje considerado um fator preditivo para a deterioração das propriedades neuromusculares. Além disso, Ohtake et al. (2018) acrescenta que pacientes que sobrevivem a doenças críticas acabam por experimentar déficits na função cognitiva e física, as quais podem persistir por anos após a permanência inicial na UTI.

A deficiência adquirida após a UTI está associada à redução da qualidade de vida relacionada à saúde e às AVDs. O estudo realizado por Trevissón-Redondo et al. (2021) mostra uma redução significativa na qualidade das AVDs entre a população idosa institucionalizada em duas casas de repouso imediatamente antes de contrair e após se recuperar da COVID19, e reitera que a idade estava intimamente relacionada com a perda da capacidade de realizar as AVDs. 
Johnson et al. (2021) em seu estudo cujo objetivo foi examinar a relação da frequência e duração da visita de fisioterapia no hospital com o estado de mobilidade dos pacientes na alta, não observaram nenhuma interação estatisticamente significativa de efeitos entre a frequência dos atendimentos de fisioterapia com as características dos pacientes. Eles observaram que com 10 minutos adicionais de exercícios físicos pacientes do sexo feminino foram mais propensas a alta para casa quando comparadas aos homens. Por outro lado, este estudo não constatou que maior frequência de atendimento fisioterapêutico estão significativamente associados a um melhor status de mobilidade na alta hospitalar. Mesmo assim, os autores sugerem que qualquer paciente com COVID-19 tratado com fisioterapia deve ter visitas com mais frequência e por períodos mais longos para alcançar maior mobilidade.

Tais achados são consistentes com os de Peiris et al. (2018) que demonstraram que o tratamento fisioterapêutico mais prolongado, melhora a funcionalidade dos idosos, principalmente na realização das AVDs e tarefas de autocuidado. Curci et al. (2021) ratifica e enfoca o papel da reabilitação precoce em pacientes com COVID-19 nos estágios iniciais da doença após a alta, afirmando que uma intervenção reabilitadora interdisciplinar pode neutralizar a deficiência e as complicações da doença aumentando a recuperação da funcionalidade dos idosos. Gwak e Hwang (2021), mostrou em seu estudo que o tratamento de reabilitação para pacientes com COVID-19 hospitalizados é eficaz para a recuperação funcional e pode ser realizado com segurança na UTI e na enfermaria geral, com equipamentos de proteção adequados e políticas de prevenção.

Dentre as estratégias de reabilitação, os artigos incluídos nesta revisão mencionam condutas que já são utilizadas antes mesmo da pandemia, como por exemplo, para os idosos que necessitaram de hospitalização, foram realizadas técnicas de mobilização precoce à beira leito, treino de equilíbrio, intervenções dietéticas, aconselhamento psicológico, acompanhamento terapêutico domiciliar após a alta hospitalar. Entretanto, não houve menção de estratégias consistentes para idosos que sofreram com o isolamento social, todavia há programas de exercícios de telessaúde e tecnologia móvel sendo desenvolvidos, para ajudar a manter a mobilidade.

Algumas limitações puderam ser encontradas em nosso estudo: estudos que tiveram diferenças nas amostras, intervenções, comparadores e definição dos desfechos, além da não realização de pesquisas em outros bancos de dados e a inclusão de artigos publicados apenas entre 2020 e 2021, nos idiomas português e inglês, que apresentassem texto para leitura na íntegra.

\section{Considerações Finais}

O presente estudo apontou efeitos deletérios da pandemia da COVID-19 à população idosa. Embora seja cedo para estimar as implicações mais concretas da pandemia nessa população, a literatura já apresenta algumas consequências negativas da COVID-19, bem como os impactos do isolamento social na saúde integral dos idosos que incluem desempenho físico prejudicado, piora da fragilidade, disfunção cognitiva e dependência funcional para a realização das AVDs. A saúde do idoso no cenário atual demanda uma atenção mais específica que busque enfatizar não só a recuperação, como também a prevenção desses agravos à saúde. Além disso, as estratégias para alcançar esses objetivos devem ser melhor estudadas, a fim de encontrar métodos para o fornecimento de assistência de qualidade a essa população.

Tendo em vista a promoção da autonomia dos idosos, é fundamental criar programas de manutenção para AVDs em situações que envolvam doenças graves, no qual seja projetado um plano de reabilitação precoce para que um idoso que sofra da infecção possa continuar a se submeter à reabilitação, a fim de não perder a capacidade de AVDs. Essas situações pandêmicas devem ser enfrentadas de forma a minimizar o impacto que podem causar na população idosa, incluindo não somente os consequentes gastos de saúde que esta situação trará. O presente estudo fornece dados e evidências que são importantes para uma equipe multiprofissional de saúde, com o intuito de avaliar a gravidade do comprometimento das AVDs dos idosos, a fim de estabelecer um plano de reabilitação imediata. 


\section{Referências}

Arazi, H., \& Eghbali, E. (2018). Osteosarcopenia and Physical Activity. Annals of sports medicine and research, 5 (1130), 3-6. https://www.jscimedcentral.com/SportsMedicine/sportsmedicine-5-1130.pdf.

Belli, S., Balbi, B., Prince, I., Cattaneo, D., Masocco, F., Zaccaria, S., Bertalli, L., Cattini, F., Lomazzo, A., Dal Negro, F., Giardini, M., Franssen, F., Janssen, D., \& Spruit, M. A. (2020). Low physical functioning and impaired performance of activities of daily life in COVID-19 patients who survived hospitalisation. The European respiratory journal, 56(4), 2002096. https://doi.org/10.1183/13993003.02096-2020

Bull, F. C., Al-Ansari, S. S., Biddle, S., Borodulin, K., Buman, M. P., Cardon, G., Carty, C., Chaput, J. P., Chastin, S., Chou, R., Dempsey, P. C., DiPietro, L., Ekelund, U., Firth, J., Friedenreich, C. M., Garcia, L., Gichu, M., Jago, R., Katzmarzyk, P. T., Lambert, E., \& Willumsen, J. F. (2020). World Health Organization 2020 guidelines on physical activity and sedentary behaviour. British journal of sports medicine, 54(24), 1451-1462. https://doi.org/10.1136/bjsports-2020-102955

CDC COVID-19. (2020). Contagens provisórias. Atualizações semanais por meio de características demográficas e geográficas selecionadas. Centro Nacional de Estatísticas de Saúde. https://www.cdc.gov/nchs/nvss/vsrr/covid_weekly/index.htm.

Cunningham, C., \& O' Sullivan, R. (2020). Why physical activity matters for older adults in a time of pandemic. European review of aging and physical activity: official journal of the European Group for Research into Elderly and Physical Activity, 17, 16. https://doi.org/10.1186/s11556-020-00249-3

Curci, C., Pisano, F., Bonacci, E., Camozzi, D. M., Ceravolo, C., Bergonzi, R., De Franceschi, S., Moro, P., Guarnieri, R., Ferrillo, M., Negrini, F., \& de Sire, A. (2020). Early rehabilitation in post-acute COVID-19 patients: data from an Italian COVID-19 Rehabilitation Unit and proposal of a treatment protocol. European journal of physical and rehabilitation medicine, 56(5), 633-641. https://doi.org/10.23736/S1973-9087.20.06339-X

Fan, E., Cheek, F., Chlan, L., Gosselink, R., Hart, N., Herridge, M. S., Hopkins, R. O., Hough, C. L., Kress, J. P., Latronico, N., Moss, M., Needham, D. M., Rich, M. M., Stevens, R. D., Wilson, K. C., Winkelman, C., Zochodne, D. W., \& Ali, N. A., ATS. (2014) Committee on ICU-acquired Weakness in Adults, \& American Thoracic Society. An official American Thoracic Society Clinical Practice guideline: the diagnosis of intensive care unit-acquired weakness in adults. American journal of respiratory and critical care medicine, 190(12), 1437-1446. https://doi.org/10.1164/rccm.201411-2011ST

Fernandez-Alonso, L., Muñoz-García, D., \& La Touche, R. (2016). The level of physical activity affects the health of older adults despite being active. Journal of exercise rehabilitation, 12(3), 194-201. https://doi.org/10.12965/jer.1632566.283

Gwak, D. W., \& Hwang, J. M. (2021). Step-by-step inpatient rehabilitation for critical illness after coronavirus disease 2019: A CARE-compliant case report. Medicine, 100(23), e26317. https://doi.org/10.1097/MD.0000000000026317

Hall, G., Laddu, D. R., Phillips, S. A., Lavie, C. J., \& Arena, R. (2021). A tale of two pandemics: How will COVID-19 and global trends in physical inactivity and sedentary behavior affect one another? Progress in cardiovascular diseases, 64, 108-110. https://doi.org/10.1016/j.pcad.2020.04.005

Herridge, M. S., Chu, L. M., Matte, A., Tomlinson, G., Chan, L., Thomas, C., Friedrich, J. O., Mehta, S., Lamontagne, F., Levasseur, M., Ferguson, N. D., Adhikari, N. K., Rudkowski, J. C., Meggison, H., Skrobik, Y., Flannery, J., Bayley, M., Batt, J., Santos, C. D., \& Abbey, S. E. (2016). Canadian Critical Care Trials Group. The RECOVER Program: Disability Risk Groups and 1-Year Outcome after 7 or More Days of Mechanical Ventilation. American journal of respiratory and critical care medicine, 194(7), 831-844. https://doi.org/10.1164/rccm.201512-2343OC

Jain, E., Harmon, E. Y., \& Sonagere, M. B. (2021). Functional outcomes and post-discharge care sought by patients with COVID-19 compared to matched controls after completing inpatient acute rehabilitation. $P M \&$ \&: the journal of injury, function, and rehabilitation, 13(6), 618-625. https://doi.org/10.1002/pmrj.12607

Johnson, J. K., Lapin, B., Green, K., \& Stilphen, M. (2021). Frequency of Physical Therapist Intervention Is Associated With Mobility Status and Disposition at Hospital Discharge for Patients With COVID-19. Physical therapy, 101(1), pzaa181. https://doi.org/10.1093/ptj/pzaa181

Kato, M., Ono, S., Seko, H., Tsukamoto, T., Kurita, Y., Kubo, A., Omote, T., \& Omote, S. (2021). Trajectories of frailty, physical function, and physical activity levels in elderly patients with heart failure: impacts of interruption and resumption of outpatient cardiac rehabilitation due to COVID-19. International journal of rehabilitation research. Internationale Zeitschrift fur Rehabilitationsforschung. Revue internationale de recherches de readaptation, 44(3), 200204. https://doi.org/10.1097/MRR.0000000000000473

Martins Van Jaarsveld G. (2020). The Effects of COVID-19 Among the Elderly Population: A Case for Closing the Digital Divide. Frontiers in psychiatry, 11, 577427. https://doi.org/10.3389/fpsyt.2020.577427

Mello, M.T., Boscolo, R.A., Esteves, A.M, \& Tufik, S. (2005). O exercício físico e os aspectos psicobiológicos. Revista brasileira de medicina do esporte, 11(3), 203-207. https://doi.org/10.1590/S1517-86922005000300010

Moro, T., \& Paoli, A. (2020). When COVID-19 affects muscle: effects of quarantine in older adults. European journal of translational myology, $30(2), 9069$. https://doi.org/10.4081/ejtm.2019.9069

Ohtake, P. J., Lee, A. C., Scott, J. C., Hinman, R. S., Ali, N. A., Hinkson, C. R., Needham, D. M., Shutter, L., Smith-Gabai, H., Spires, M. C., Thiele, A., Wiencek, C., \& Smith, J. M. (2018). Physical Impairments Associated With Post-Intensive Care Syndrome: Systematic Review Based on the World Health Organization's International Classification of Functioning, Disability and Health Framework. Physical therapy, 98(8), 631-645. https://doi.org/10.1093/ptj/pzy059

Parry, S. M., \& Puthucheary, Z. A. (2015). The impact of extended bed rest on the musculoskeletal system in the critical care environment. Extreme physiology \& medicine, 4, 16. https://doi.org/10.1186/s13728-015-0036-7

Peiris, C. L., Shields, N., Brusco, N. K., Watts, J. J., \& Taylor, N. F. (2018). Additional Physical Therapy Services Reduce Length of Stay and Improve Health Outcomes in People With Acute and Subacute Conditions: An Updated Systematic Review and Meta-Analysis. Archives of physical medicine and rehabilitation, 99(11), 2299-2312. https://doi.org/10.1016/j.apmr.2018.03.005 
Roman, A. R. Friedlander, M. R. (1998) Revisão integrativa de pesquisa aplicada à enfermagem. Cogitare Enfermagem, 3 (2), 109-112. http://dx.doi.org/10.5380/ce.v3i2.44358

Santini, Z. I., Jose, P. E., York Cornwell, E., Koyanagi, A., Nielsen, L., Hinrichsen, C., Meilstrup, C., Madsen, K. R., \& Koushede, V. (2020). Social disconnectedness, perceived isolation, and symptoms of depression and anxiety among older Americans (NSHAP): a longitudinal mediation analysis. The Lancet. Public health, 5(1), e62-e70. https://doi.org/10.1016/S2468-2667(19)30230-0

Sepúlveda-Loyola, W., Rodríguez-Sánchez, I., Pérez-Rodríguez, P., Ganz, F., Torralba, R., Oliveira, D. V., \& Rodríguez-Mañas, L. (2020). Impact of Social Isolation Due to COVID-19 on Health in Older People: Mental and Physical Effects and Recommendations. The journal of nutrition, health \& aging, 24(9), 938-947. https://doi.org/10.1007/s12603-020-1469-2

Shahid, Z., Kalayanamitra, R., McClafferty, B., Kepko, D., Ramgobin, D., Patel, R., Aggarwal, C. S., Vunnam, R., Sahu, N., Bhatt, D., Jones, K., Golamari, R., \& Jain, R. (2020). COVID-19 and Older Adults: What We Know.Journal of the American Geriatrics Society, 68(5), 926-929. https://doi.org/10.1111/jgs.16472

Shinohara, T., Saida, K., Tanaka, S., \& Murayama, A. (2021). Actual Frailty Conditions and Lifestyle Changes in Community-Dwelling Older Adults Affected by Coronavirus Disease 2019 Countermeasures in Japan: A Cross-Sectional Study. SAGE open nursing, 7 , 23779608211025117. https://doi.org/10.1177/23779608211025117

Silva, L. R. B., Seguro, C. S., Oliveira, C. G. A, Santos, P. O. S., Oliveira, J. C. M., Souza Filho, L. F. M., de Paula Júnior, C. A., Gentil, P., Rebelo, A. C. S. (2020). Physical Inactivity Is Associated With Increased Levels of Anxiety, Depression, and Stress in Brazilians During the COVID-19 Pandemic: A CrossSectional Study. Frontiers in psychiatry, 11, 1257. https://doi.org/10.3389/fpsyt.2020.565291

Steardo, L., Steardo, L., Jr, Zorec, R., \& Verkhratsky, A. (2020). Neuroinfection may contribute to pathophysiology and clinical manifestations of COVID19. Acta physiologica (Oxford, England), 229(3), e13473. https://doi.org/10.1111/apha.13473

Tomioka, K., Kurumatani, N., Hosoi, H. (2017). Association between Social Participation and 3-Year Change in Instrumental Activities of Daily Living in Elderly Residents in the Community. Journal american geriatrics society, 65(1), 107-113. https://doi.org/10.1111/jgs.14447

Trevissón-Redondo, B., López-López, D., Pérez-Boal, E., Marqués-Sánchez, P., Liébana-Presa, C., Navarro-Flores, E., Jiménez-Fernández, R., Corral-Liria, I., Losa-Iglesias, M., \& Becerro-de-Bengoa-Vallejo, R. (2021). Use of the Barthel Index to Assess Activities of Daily Living before and after SARS-COVID 19 Infection of Institutionalized Nursing Home Patients. International journal of environmental research and public health, 18(14), 7258. https://doi.org/10.3390/ijerph18147258

Van Gerwen, M., Alsen, M., Little, C., Barlow, J., Genden, E., Naymagon, L., \& Tremblay, D. (2021). Risk factors and outcomes of COVID-19 in New York City; a retrospective cohort study. Journal of medical virology, 93(2), 907-915. https://doi.org/10.1002/jmv.26337

World Health Organization. (2021). Weekly Operational Update on COVID-19. https://www.who.int/publications/m/item/weekly-operational-update-oncovid-19---26-january-2021.

Zerah, L., Baudouin, É., Pépin, M., Mary, M., Krypciak, S., Bianco, C., Roux, S., Gross, A., Toméo, C., Lemarié, N., Dureau, A., Bastiani, S., Ketz, F., Boully, C., de Villelongue, C., Romdhani, M., Desoutter, M. A., Duron, E., David, J. P.,Thomas, C., \& Boddaert, J. (2021). Clinical Characteristics and Outcomes of 821 Older Patients With SARS-Cov-2 Infection Admitted to Acute Care Geriatric Wards. The journals of gerontology. Series A, Biological sciences and medical sciences, 76(3), e4-e12. https://doi.org/10.1093/gerona/glaa210 